\title{
Single top-quark production with the Matrix Element Method in next-to-leading order accuracy
}

\author{
T. Martini* and P. Uwer \\ Humboldt-Universität zu Berlin, Institut für Physik, Newtonstraße 15, 12489 Berlin, Germany \\ E-mail: Till.Martini@physik.hu-berlin.de, \\ Peter.Uwerephysik.hu-berlin.de
}

\begin{abstract}
Single top-quark production offers a unique laboratory for precision tests of the Standard Model and searches of possible extensions. Furthermore, assuming the Standard Model, single top-quark production can be used to determine top-quark related couplings. For precise determinations of parameters like the electroweak gauge couplings or the mass of the top quark, efficient, unbiased, and theoretically unambiguous analysis methods are needed. Within this context, the Matrix Element Method (MEM) has been established in hadron collider analyses due to its possibility to top out at utilising the information available in experimental data. However, so far it has mostly been applied in Born approximation only. We discuss the extension to next-to-leading order (NLO) accuracy. As a necessary prerequisite we introduce an efficient method to calculate NLO QCD weights for jet events. As proof of concept and representative example we use the MEM at NLO to reproduce the top-quark mass in a toy experiment where we treat single top-quark events generated at NLO accuracy as pseudo-data. This is the first application of the MEM at NLO accuracy to the hadronic production of jets originating from coloured final state partons. We observe that analysing NLO events with Born likelihoods can introduce a pronounced bias in the extracted mass which would require significant calibration with associated uncertainties. Although we focus on parameter determinations, the methods presented here can also be used to search for new physics using likelihood ratios.
\end{abstract}

XXV International Workshop on Deep-Inelastic Scattering and Related Subjects 3-7 April 2017

University of Birmingham, UK

\footnotetext{
* Speaker.
} 


\section{The MEM in a nutshell}

For the collision $A+B \rightarrow a_{1}\left(p_{1}\right)+a_{2}\left(p_{2}\right)+\ldots+a_{n}\left(p_{n}\right)$ the differential cross section $\frac{d \sigma}{d R_{n}\left(p_{1}, \ldots, p_{n}\right)} \propto\left|\mathscr{M}\left(p_{1}, \ldots, p_{n}\right)\right|^{2} d R_{n}$ is a measure for the probability to observe the final state in the infinitesimal phase space region $d R_{n}=(2 \pi)^{4} \delta\left(P-\sum_{i=1}^{n} p_{i}\right) \prod_{j=1}^{n} \frac{d^{3} p_{j}}{(2 \pi)^{3} 2 E_{j}}$ located at $\left(p_{1}, \ldots, p_{n}\right)$. By collecting generic partonic final state variables (e.g. $\left.E_{i}, \eta_{j}, \ldots\right)$ in $\vec{y}$ we can generalise the differential cross section to $\frac{d \sigma}{d R_{n}\left(p_{1}, \ldots, p_{n}\right)} \rightarrow \frac{d \sigma_{n}}{d \vec{y}}$. Experimentally, only hadronic variables $\vec{x}$ instead of partonic variables $\vec{y}$ are observed. The correspondence between $\vec{y}$ and $\vec{x}$ is given by the so-called Transfer Function $W(\vec{x}, \vec{y})$ which models the probability to observe a partonic event $\vec{y}$ as a hadronic event $\vec{x}$. These Transfer Functions have to be determined by experiments through detector simulations. In the following, we will set them to $\delta$-functions as a first approximation assuming an ideal detector. Non-trivial transfer functions require additional integrations over the partonic variables but do not pose a principle restriction for the method presented here. With these deliberations we can calculate a model dependent likelihood for having measured a specific event sample $\left\{\vec{x}_{i}\right\}$ as the product of the individual probabilities for each event in the sample

$$
\mathscr{L}(\omega)=\prod_{i} \frac{1}{\sigma}(\omega) \int d \vec{y} \frac{d \sigma(\omega)}{d \vec{y}} \underbrace{W\left(\vec{x}_{i}, \vec{y}\right)}_{=\delta\left(\vec{x}_{i}-\vec{y}\right)}=\prod_{i} \frac{1}{\sigma(\omega)} \frac{d \sigma(\omega)}{d \vec{x}_{i}} .
$$

This likelihood is a function of model parameter(s) $\omega$ entering the cross section calculation within a given model. Maximising the likelihood for the event sample at hand with respect to $\omega$ yields an estimator $\widehat{\omega}$ for the model parameter(s): $\mathscr{L}(\widehat{\omega})=\sup _{\omega} \mathscr{L}(\omega)$. Because all information from the event is used in the matrix element when evaluating the likelihood the MEM ideally results in the most efficient estimator. Pioneered at the Tevatron (see e.g. ref. [1, 2, 3]) the MEM has been widely used since. However, the experimental analysis is restricted to Born approximation so far.

\section{Extending the MEM to NLO}

In the past some effort was put into including higher order corrections into the MEM: The effect of QCD radiation was studied in ref. [4]. The hard Born matrix element is combined with a parton shower in ref. [5]. Full NLO corrections to the production of uncoloured objects were introduced in ref. [6] and applied in ref. [7]. Ref. [8] investigates the inclusion of hadronic jet production by mapping NLO and LO jets with a boost along the beam axis to balance the transverse momentum. A complete algorithm based on modified $(3 \rightarrow 2)$ clustering prescriptions to extend the MEM to NLO for arbitrary initial and final states was published in ref. [9] . Ref. [10] suggests to retain the $(2 \rightarrow 1)$ clustering prescriptions by numerically solving a system of non-linear equations.

When calculating cross sections new features enter at NLO: Infrared (IR) divergences in virtual and real contributions have to be mutually cancelled and the $n+1$ particle phase space of the real corrections introduces a non-trivial mapping of parton momenta $\left(p_{1}, \ldots, p_{n+1}\right)$ to the jet momenta $\left(\widetilde{J}_{1}\left(p_{1}, \ldots, p_{n+1}\right), \ldots, \widetilde{J}_{n}\left(p_{1}, \ldots, p_{n+1}\right)\right)$. Extending the likelihood to NLO accuracy using

$$
\mathscr{L}^{\mathrm{NLO}}(\omega)=\left.\prod_{i} \frac{1}{\sigma_{n \text {-jet }}^{\mathrm{NLO}}(\omega)}\left(\frac{d \sigma_{n \rightarrow n \text {-jet }}^{\mathrm{B}+\mathrm{V}}(\omega)}{d R_{n}\left(J_{1}, \ldots, J_{n}\right)}+\frac{d \sigma_{n+1 \rightarrow n \text {-jet }}^{\mathrm{R}}(\omega)}{d R_{n}\left(\widetilde{J}_{1}, \ldots, \widetilde{J}_{n}\right)}\right)\right|_{\text {event } i}
$$


where $d \sigma_{n \rightarrow n \text {-jet }}^{\mathrm{B}+\mathrm{V}}\left(d \sigma_{n+1 \rightarrow n \text {-jet }}^{\mathrm{R}}\right)$ denotes the sum of Born and virtual (real) contributions, three requirements have to be fulfilled:

1. Both contributions are separately IR divergent. To ensure a point-wise cancellation within phase space both contributions have to be evaluated for the same jet momenta: $J_{i}=\widetilde{J_{i}}$.

2. In the real contribution the clustering of $n+1$ partons to $n$ jets introduces $\delta$-functions $\delta\left(J_{i}-\right.$ $\left.\widetilde{J}_{i}\left(p_{1}, \ldots, p_{n+1}\right)\right)$ in the phase space integration which render a straight-forward numerical integration problematic.

3. To evaluate the Born and virtual matrix elements for the jet momenta the clustered jets have to be on-shell: $J_{i}^{2}=m_{i}^{2}$ and respect momentum conservation: $J_{1}+\ldots+J_{n}=p_{1}+\ldots+p_{n+1}$ at the same time.

To meet all three requirements at once we propose to replace the common $(2 \rightarrow 1)$ clustering prescriptions in jet algorithms with $(3 \rightarrow 2)$ clustering prescriptions inspired by the Catani-Seymour dipole subtraction method (see ref. [11]). Using these modified jet algorithms allows to factorise the phase space $d R_{n+1}\left(p_{1}, \ldots, p_{n+1}\right)=d R_{n}\left(\widetilde{J_{1}}, \ldots, \widetilde{J_{n}}\right) d R_{\text {unres }}(\Phi)$ and offers the possibility to consistently define a differential jet cross section (jet event weight) at NLO accuracy :

$$
\frac{d \sigma_{n-\mathrm{jet}}^{\mathrm{NLO}}(\omega)}{d R_{n}\left(J_{1}, \ldots, J_{n}\right)}=\frac{d \sigma_{n \rightarrow n \text {-jet }}^{\mathrm{B}+\mathrm{V}}(\omega)}{d R_{n}\left(J_{1}, \ldots, J_{n}\right)}+\left.\int d R_{\mathrm{unres}}(\Phi) \frac{d \sigma_{n+1 \rightarrow n \text {-jet }}^{\mathrm{R}}(\omega)}{d R_{n+1}\left(p_{1}, \ldots, p_{n+1}\right)}\right|_{p_{i}=p_{i}\left(J_{1}, \ldots, J_{n}, \Phi\right)} .
$$

For details we refer to [9]. To cancel IR divergences in both terms, we employ the Phase Space Slicing (PSS) method. The NLO jet event weight can be used to generate unweighted NLO jet events or to construct the NLO likelihood for the MEM.

\section{Validation}

To validate the real phase space generation and the generation of unweighted events we reproduce NLO jet distributions calculated using a conventional parton level Monte-Carlo generator with a $(3 \rightarrow 2)$ jet algorithm. The $s$ - and $t$-channel production of single top quarks in association with a light jet at the LHC $(\sqrt{s}=13 \mathrm{GeV})$ is studied: $p p \rightarrow t j(X)$. We study the exclusive (additional jet activity $X$ is vetoed) and the inclusive (additional jet activity $X$ is allowed) case. For simplicity we do not consider the decay of the top quark but treat them as tagged top jets. Details on the calculation using Phase Space Slicing to cancel the IR divergences can be found in ref. [12].
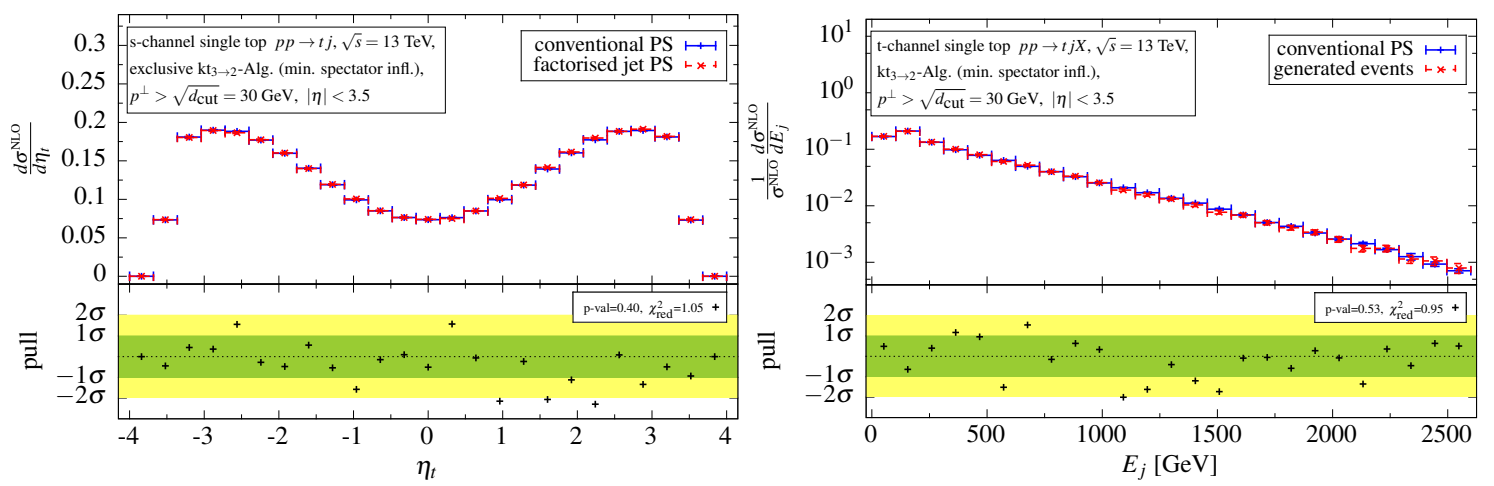

Figure 1: Validation of phase space generation (left) and event generation (right). 
The left hand side of fig. 1 shows the comparison of the pseudo rapidity distribution of the toptagged jet in the exclusive $s$-channel obtained using a conventional parton-level MC (solid blue) and the method outlined in eq. 2.1 (red dashed). The right hand side compares a histogram of the energy of the light jet filled with unweighted inclusive t-channel NLO events (red dashed) generated using again eq. 2.1. These results are compared to the distribution obtained from a conventional parton-level MC (solid blue). In both cases, we find perfect agreement (see pull distribution at the bottom of the plots). Analogous comparisons of other observables for the exclusive and inclusive $s$ - and $t$-channel show similar results and can be found in ref. [13].

\section{Application}

The NLO jet event weight eq. 2.1 can be used in the MEM at NLO to recover the input value of the top mass $m_{t}^{\text {true }}=173.2 \mathrm{GeV}$ from the generated NLO jet events $\vec{x}_{i}=\left(\eta_{t}, E_{j}, \eta_{j}, \phi_{j}\right)_{i}$ e.g.:

$$
\mathscr{L}^{\mathrm{NLO}}\left(m_{t}\right)=\prod_{i}^{N} \mathscr{L}^{\mathrm{NLO}}\left(\vec{x}_{i} \mid m_{t}\right)=\left.\left(\frac{1}{\sigma^{\mathrm{NLO}}\left(m_{t}\right)}\right)^{N} \prod_{i=1}^{N}\left(\frac{E_{j}^{2} \cosh \left(\eta_{t}\right)}{2 s E_{t} \cosh ^{3}\left(\eta_{j}\right)} \frac{d \sigma^{\mathrm{NLO}}}{d R_{2}\left(J_{t}, J_{j}\right)}\left(m_{t}\right)\right)\right|_{\text {event } i}
$$

Fig. 2 shows the extraction of the top-quark mass with NLO (reddish) and Born likelihoods (bluish) with simultaneous variations of the renormalisation and factorisation scales by factors $\frac{1}{2}$ (subscript) and 2 (superscript) as a measure for higher order effects. The estimators are extracted as the minima of the negative logarithm of the likelihoods ('Log-Likelihoods') by a parabola fit.
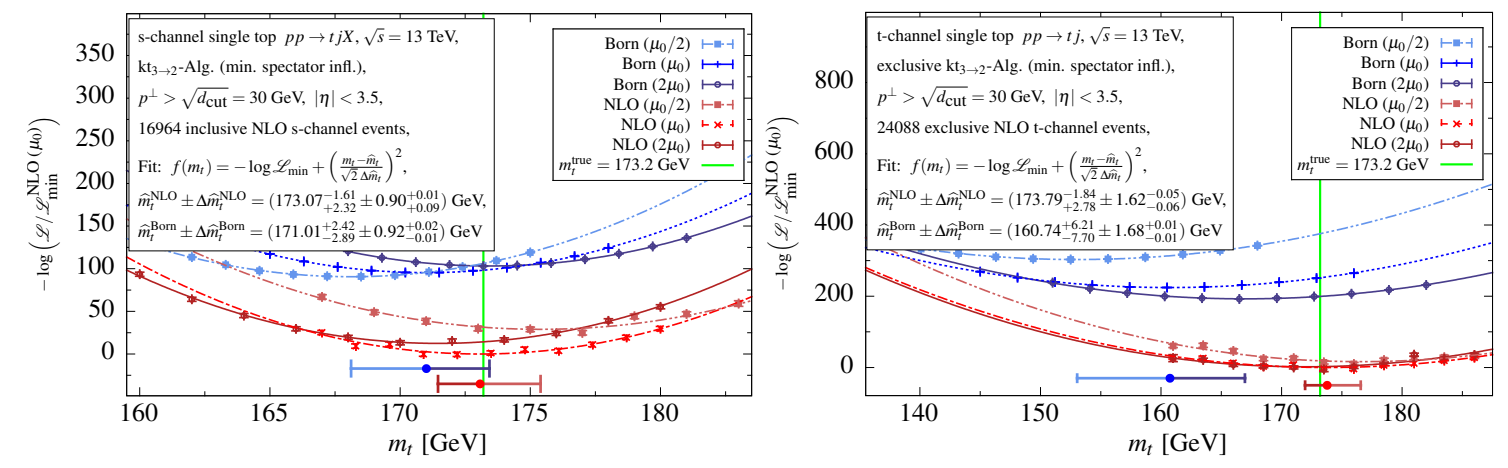

Figure 2: Extraction of the top mass with MEM at NLO or Born accuracy.

The left hand side of fig. 2 shows that around 17000 inclusive $s$-channel NLO events allow a precise mass determination with a relative statistical uncertainty of $\widehat{m}_{t} / \Delta \widehat{m}_{t}=0.5 \%$. Applying the MEM at NLO perfectly recovers the input value $m_{t}^{\text {true }}$. However, using Born likelihoods on the same events introduces a bias in the estimator $\widehat{m}_{t}^{\text {Born }}$ of $-2.2 \mathrm{GeV}$. This bias is covered by the scale variations shifting the Born estimator by $-2.9 \mathrm{GeV}$ and $+2.4 \mathrm{GeV}$. The impact of the scale variation on the NLO estimator is reduced to $-1.6 \mathrm{GeV}$ and $+2.3 \mathrm{GeV}$. In contrast, the right hand side of fig. 2 demonstrates that analysing NLO events with Born likelihoods can result in large biases in the estimator which are not necessarily covered by the scale variations. Applying the MEM at NLO to exclusive $t$-channel NLO events again perfectly reproduces $m_{t}^{\text {true }}$ while the Born estimator is shifted by $-12.5 \mathrm{GeV}$. Scale variations shift the Born estimator by $-7.7 \mathrm{GeV}$ and $+6.2 \mathrm{GeV}$. In the NLO analysis the impact of the scale variations is significantly reduced to $-1.8 \mathrm{GeV}$ and $+2.8 \mathrm{GeV}$. 
In the Born analysis the simultaneous variations of the renormalisation and factorisation scales by factors $\frac{1}{2}$ and 2 do not give a reliable estimate of the NLO effects. The large bias observed in the Born estimator in the right hand side of fig. 2 would require a significant calibration of the MEM which introduces related uncertainties. It should be noted that the renormalisation scheme is welldefined in the MEM at NLO. The extracted top-quark mass equates to the pole mass. For a more in-depth study of single top-quark production with the MEM at NLO we refer to [13].

\section{Conclusion}

We have presented an algorithm to extend the MEM to NLO accuracy. We have illustrated the method using single top-quark production as an example. We find a significant improvement compared to leading-order predictions. In particular, the calibration required when the MEM is applied in leading-order is reduced. Although not illustrated here the method can also be used for new physics searches using likelihood ratios.

\section{References}

[1] D0 collaboration, B. Abbott et al., Measurement of the Top Quark Mass in the Dilepton Channel, Phys. Rev. D60 (1999) 052001, [hep-ex/9808029].

[2] D0 collaboration, V. M. Abazov et al., A precision measurement of the mass of the top quark, Nature 429 (2004) 638-642, [hep-ex/ 0406031$].$

[3] CDF collaboration, A. Abulencia et al., Top quark mass measurement from dilepton events at CDF II with the matrix-element method, Phys. Rev. D74 (2006) 032009, [hep-ex/ 0605118$].$

[4] J. Alwall, A. Freitas and O. Mattelaer, The Matrix Element Method and QCD Radiation, Phys. Rev. D83 (2011) 074010, [1010.2263].

[5] D. E. Soper and M. Spannowsky, Finding physics signals with event deconstruction, Phys. Rev. D89 (2014) 094005, [1402.1189].

[6] J. M. Campbell, W. T. Giele and C. Williams, The Matrix Element Method at Next-to-Leading Order, JHEP 11 (2012) 043, [1204.4424].

[7] J. M. Campbell, R. K. Ellis, W. T. Giele and C. Williams, Finding the Higgs boson in decays to Z $\gamma$ using the matrix element method at Next-to-Leading Order, Phys. Rev. D87 (2013) 073005, [1301.7086].

[8] C. Williams, J. M. Campbell and W. T. Giele, Event-by-event weighting at next-to-leading order, PoS RADCOR2013 (2013) 037, [1311.5811].

[9] T. Martini and P. Uwer, Extending the Matrix Element Method beyond the Born approximation: Calculating event weights at next-to-leading order accuracy, JHEP 09 (2015) 083, [1506. 08798].

[10] R. Baumeister and S. Weinzierl, Matrix element method at next-to-leading order for arbitrary jet algorithms, Phys. Rev. D95 (2017) 036019, [1612 . 07252].

[11] S. Catani, S. Dittmaier, M. H. Seymour and Z. Trocsanyi, The Dipole formalism for next-to-leading order QCD calculations with massive partons, Nucl.Phys. B627 (2002) 189-265,

[12] Q.-H. Cao and C.-P. Yuan, Single top quark production and decay at next-to-leading order in hadron collision, Phys. Rev. D71 (2005) 054022, [hep-ph / 0408180 ].

[13] T. Martini and P. Uwer, in preparation, . 